\title{
Elbow Prosthesis for Partial or Total Upper Limb Replacements
}

\author{
Federico Casolo \\ Politecnico di Milano, \\ Department of Mechanics
}

Italy

\section{Introduction}

While the prosthetic systems for hands amputation are fairly common, the active prostheses for the other joints of the upper $\operatorname{limb}$ are rarer. In addition to commercial reasons undoubtedly related to the low number of trans-humeral amputations than the hands amputations and to the exiguous number of shoulder disarticulation, technical reasons are also crucial. Among which, the electro-mechanical complexity of the system and, above all, the difficulty to design simple and "natural" motion control systems. Nevertheless active elbow and shoulder prosthetic joints can be very important for some upper limb amputees for the everyday life, for working or for other social reasons, in particular if the amputation is bilateral.

The upper limb prostheses can by functional or can have only cosmetic purposes. The functional prosthetic arms can have passive or active features.

The active artificial upper limbs can be divided into prostheses powered by body energy and prostheses powered by external energy. To the first group belong systems actuated by the motion of other body joints and systems moved by means of the arm muscles through their tendons(Chilress et al, 2001). The last, intuitive and theoretically easy to control, were somewhat popular in central Europe in mid 20th century, but they have been virtually abandoned because of critical problem both biological and mechanical.

On the contrary the upper limbs systems that transmit to the prostheses, through cables, the displacement of other body compartments are still common, and some special applications of this method are still under development. They are mostly suggested to active subjects, with adequate mobility of the other upper body structures, which want to feel a direct complete control of their artificial joints.

However, at present, most of artificial upper limbs are equipped by joints powered by external energy. This energy is in most cases electric and is stored in portable batteries but there are also examples of pressure energy stored in little gas tank mounted on the arm. An important requirement for the systems that use extra body energy is their autonomy: the stored energy must last at least for a whole day. Electrical prosthetic systems can benefit of the constant improvement of battery performance due to the rapid evolution of very popular potable products - e.g. cell. phones and computer -. 


\section{Active artificial arms motion control}

Unlike to the body powered system which are directly driven by the patient and controlled by means of his senses, the ones using extra body power require a more complex approach to the arm motion control. At present one of the most important sources of control are the e.m.g. signal of residual muscles of the subjects; the muscles used can be inside the stump or external to it. The e.m.g. signals are normally used when the independent active axes are not more than three, but generally they are actuated in series because the co-activation of two or more specific muscles generate too much mental stress for the subject: only very few and young trained subjects demonstrated to be able to drive two joints simultaneously. Therefore, in general only one degree of freedom at a time is activated by the e.m.g. signal of two muscles, one for the joint flexion and one for the extension, while previously another signal is used to select the joint to be moved. The signal to operate the serial selector can be obtained or acting on proper belts or by means of another e.m.g. source. The sequential activation of the joints produces un-physiological and ungainly movements, therefore for multi axes systems other procedures have been implemented.

The purpose is to replicate the natural approach to the arm movement in which the subject governs the hand, thinking only very marginally to the joints of the arm. In fact, our approach to the artificial arm motion only requires the knowledge of position where the amputee want to place the artificial hand, all the other factors, such as joint trajectories and motors control are automatically managed by the system and are transparent to the subject (Camposaragna et al., 2001). Therefore the arm driving problem is made of two distinct parts: to interpret the patient will and to produce the arm movement, the first of which is the most difficult. In order to interpret the patient will, many parameters can contribute alone or combined to the aim, such as: e.m.g and b.c.i.(brain computer interface) signals, besides movement of other body parts. At present a research on the most effective composition of signals collectable from the patient to interpret his/her request of hand movement is under development at Politecnico di Milano, where a new b.c.i. helmet have been recently produced. Until now we are using only the head displacement to move the artificial arm and two e.m.g. signals to open and close the hand. We already tested two algorithms to drive the arm though the head movements, only one of which, during the action, requires that the patient directs continuously the head toward the instantaneous hand target. This technique have been also implemented in a virtual reality system designed to: identify the patients eligible to the use of the artificial limb, to train the subjects to drive the prosthesis before it is available, to personalize the driving algorithms parameters and to certify the progress in the learning process (Camposaragna et al, 2001).

The same procedure is used for multi axes arm systems addressed to shoulder disarticulated subjects, as well as to trans-humeral amputees: in the latter case together with the head kinematics also the stump position must be collected. The virtual reality exercises requires exactly the same input used by the real prosthetic arm, that includes the head kinematics, the e.m.g. signals to open or close the hand and, if the forearm is present, also its kinematics. To calculate the kinematics of each monitored body segment, the last version of our system is equipped with two Inertial Motion Tracker which includes a triaxial accelerometer, a triaxial gyroscope and a gravitometer (fig. 1).

The arm mathematical model used for the computer simulation take into account the electro-mechanical characteristics of the system, included the motors torque vs. velocity ratio and the whole system mechanical efficiency. 
This allows training the subjects to develop the driving skills for his definitive artificial arm by means of a simulated arm which respond to the commands just like the prosthesis does.
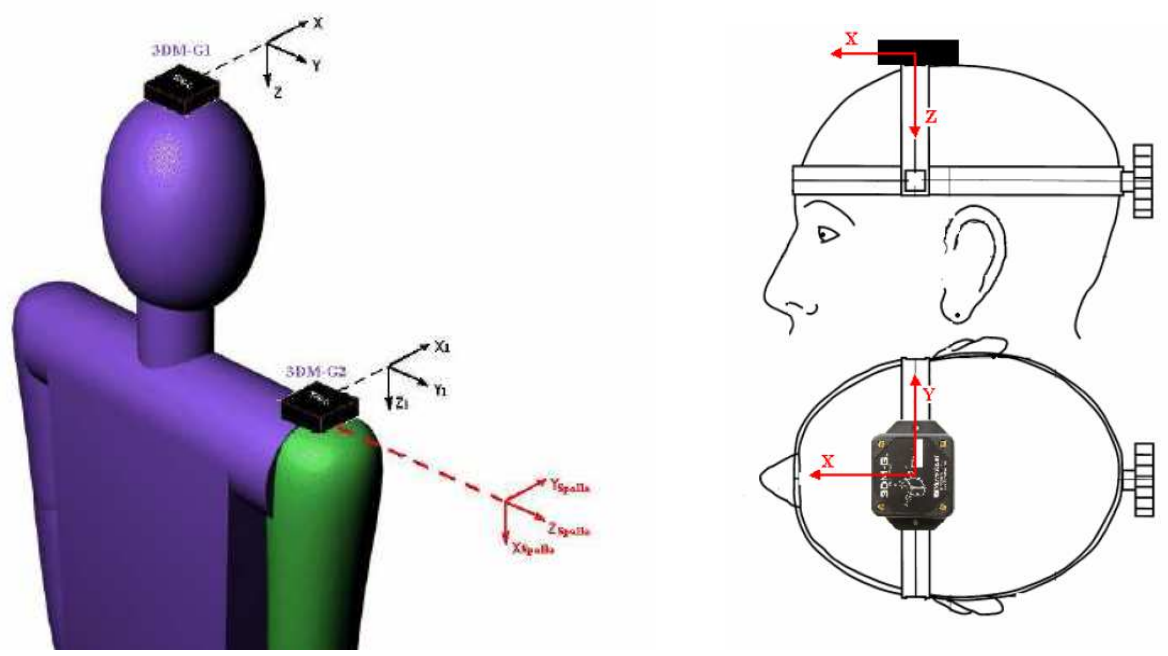

Fig. 1. Location of the Inertial Motion Trackers, with their reference axes

When the problem to extract, from the signal collected from the amputee, the kinematics of the hand target is solved, one of the main steps of the work is completed.

Next point is to define how to fill the gap between hand and target: the easiest solution would be to set the velocity inversely proportional to their distance, but with this procedure the average velocity is too slow, thus an exponential negative function have been implemented in order to increase the average velocity while keeping a low velocity near the target.

The next step is the solution of the forward dynamic problem of the arm, that is to calculate the required motor torque and to verify if it is compatible with the characteristic diagram of the motor (Legnani et al 1996). If negative, the dynamic requests are decreased in order to fulfil the motor limits.

The control system is modular: the main board calculates the target and arm kinematics, and send to the board of each motor - which is generally brushless - the setting points of velocity and position.

To decrease the computational load of the arm main board, the need of solving the trigonometric functions has been avoided by pre compiling a look-up table containing, for each square centimetre of the working volume of the hand, the corresponding angular joint coordinate of each motor of the limb. I2C protocol has been implemented for boards communication. The I2C bus physically consists of 2 active wires and a ground connection. The active wires, the serial data line, and the serial clock line, are both bidirectional (Nxp, 2007). The motors instantaneous velocity and position are calculated from the hall sensors signals. With our present approach the motor is controlled directly in velocity (fig.2) in order to be able to force also acceleration and deceleration, while the external ring is only used to check whether the position set point has been reached. 


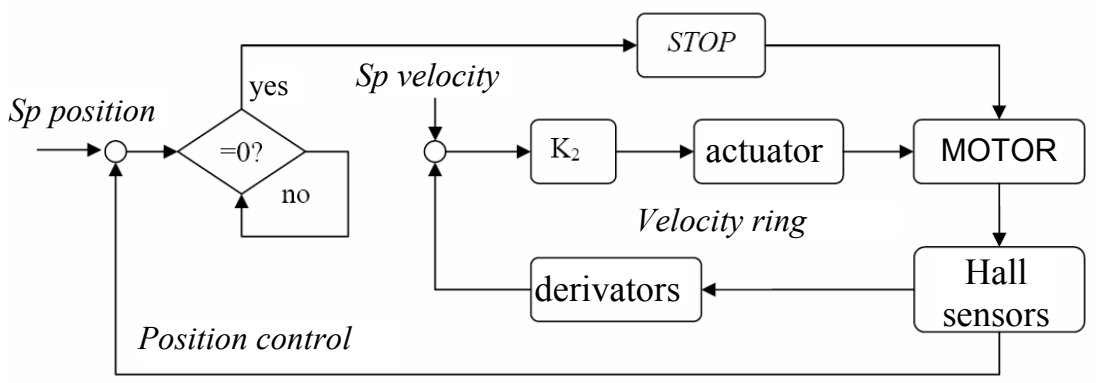

Fig. 2. Schema of the brushless motor control

\section{Prosthetic active joint efficiency: an example of elbow transmission design}

The system mechanical efficiency is a key factor of the whole project because it is strictly related to the torque required to the motor and consequently to the size and the weight of motor, of transmission and of the battery package. Also motor and transmission noise is, in general, inversely proportional to the mechanical efficiency. Most elbow prostheses of the past generation were quite inefficient because they employ small and fast DC motors coupled with transmissions made of long chains of gears. Each couple of gears waist energy for friction and the whole chain efficiency - product of the efficiencies of all the couple of gears - can assume very little values, even lover than $15 \%$. This is the case of the elbow joint (fig.3a) that our group -MSUM- used as reference for the design of new elbows (Fig.3b, 3c).

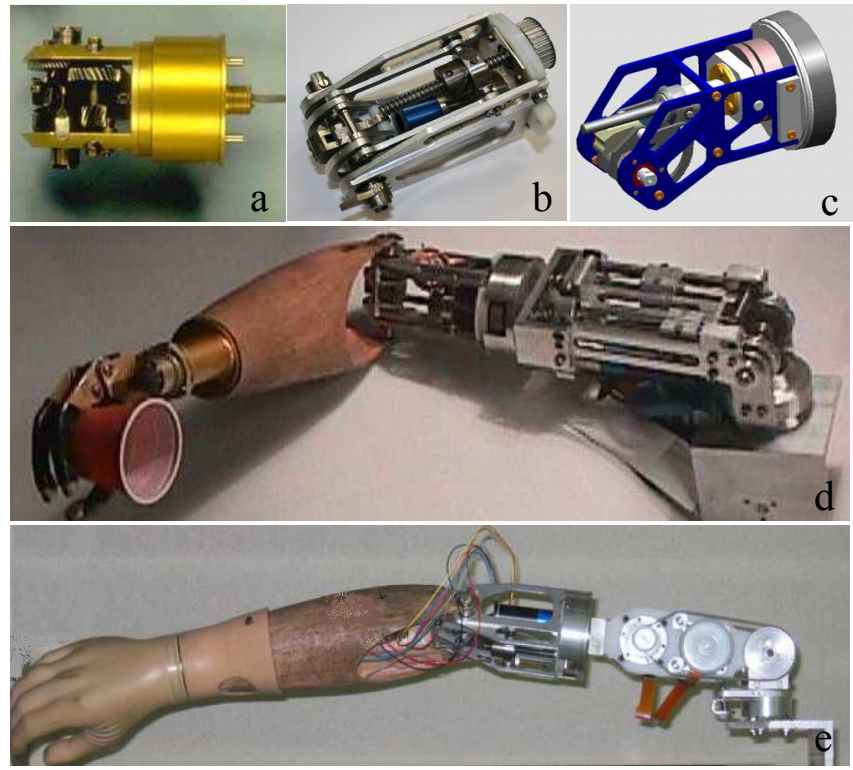

Fig. 3. a) Old elbow with a long chain of gears; b) MSUM elbow1; c) MSUM elbow2; d) Full arm with MSUM-shoulder1, MSUM-elbow2 and Otto-bock hand; e) Full arm with MSUMshoulder2, MSUM-elbow1 with Otto-bock hand and wrist. 


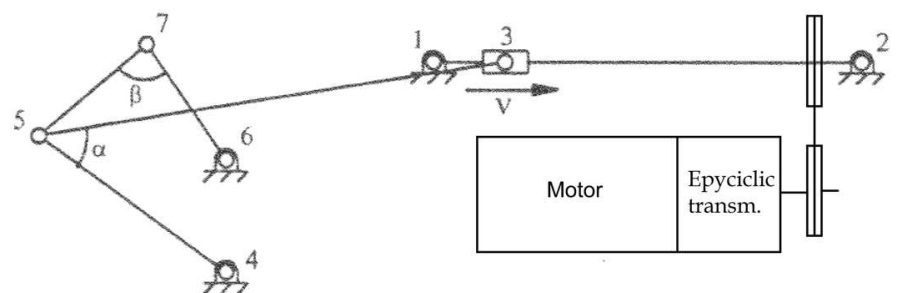

Fig. 4. Ebow1 with epicyclic transmission.

One method to increase the mechanical efficiency is to substitute, when possible, the gears by linkages with ball bearing and by screw-ball transmissions. The first elbow prosthesis designed by our MSUM group and used by patients with satisfaction, is made of a ball screw axis acting on a slider crank connected to a four bars linkage. The ball screw axis is connected to a brushless motor through an epicyclical transmission and a belt and pulley system. The measured overall mechanical efficiency of the system was increased up to $64 \%$. In the second model (Fig.3c and Fig.4) the screwball axis is oscillating and is directly connected to a pancake brushless motor. This architecture raise the efficiency to over $80 \%$. As an example, below are reported the main steps of the design and analysis procedure for the latter elbow system (Fig.5a).

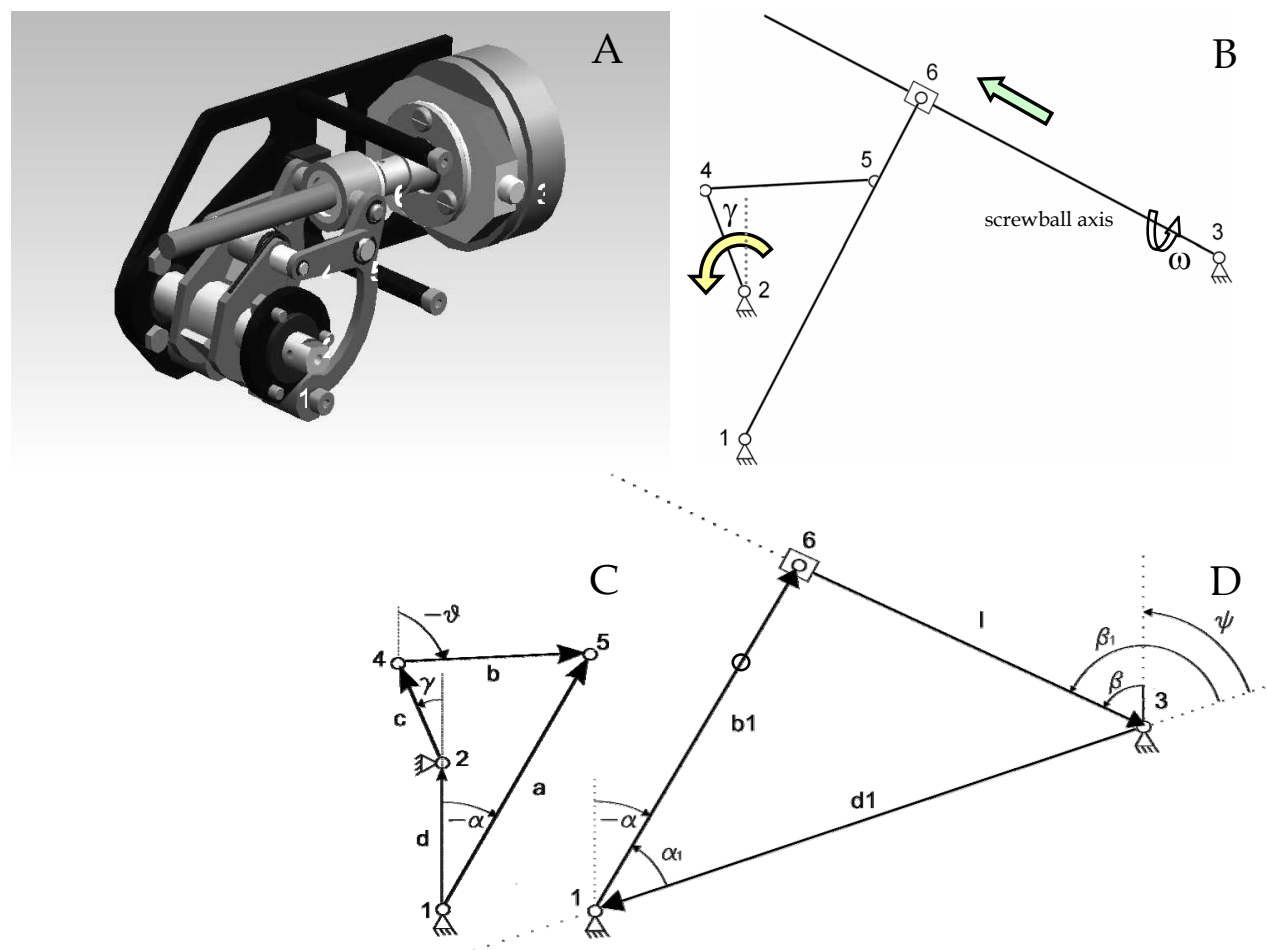

Fig. 5. a) MSUM elbow2 joint ; b) kinematic model of the full joint ; breakdown of the in two mechanisms in series with their vector representation : c) four bar linkage mechanism and d) slider-crank mechanism with the oscillating bar that is also the axis of the pancake motor. 
Considering only the four bars linkage (fig. 5 c) its kinematics can be analyzed as follows:

$$
\begin{aligned}
& a \cdot e^{i \alpha}-b \cdot e^{i \theta}-c \cdot e^{i \gamma}-d=0 \\
& \left\{\begin{array}{c}
b \cos \theta=a \cos \alpha-c \cos \gamma-d \\
\quad b \sin \theta=a \sin \alpha-c \sin \gamma
\end{array}\right. \\
& a^{2}-b^{2}+c^{2}+d^{2}-2 a c \cos \gamma \cos a-2 a c \sin \gamma \sin a-2 a d \cos a+2 c d \cos \gamma=0
\end{aligned}
$$

and grouping to simplify the expressions

$$
\left\{\begin{array}{c}
A=-2 a c \sin \gamma \\
B=-2 a d-2 a c \cos \gamma \\
C=a^{2}-b^{2}+c^{2}+d^{2}+2 c d \cos \gamma \\
D=\sqrt{A^{2}+B^{2}-C^{2}}
\end{array}\right.
$$

we obtain, with few passages, the expressions of $\alpha$ and $\theta$ as function of $\gamma$

$$
\begin{aligned}
& \alpha=\arcsin \left(-\frac{A C-B D}{A^{2}+B^{2}}\right) \\
& \theta=\arctan \left(\frac{c \sin \gamma-a \sin \alpha}{d+c \cos \gamma-a \cos \alpha}\right)
\end{aligned}
$$

Similarly, considering only the slider crank oscillating system (Fig.5d):

$$
\begin{aligned}
& b_{1} \cdot e^{i \alpha_{1}}-l \cdot e^{i \beta_{1}}-d_{1}=0 \\
& \left\{\begin{array}{c}
l=\sqrt{b_{1}^{2}+d_{1}^{2}-2 b_{1} d_{1} \cos \alpha_{1}} \\
\sin \beta_{1}=b_{1} \sin \alpha_{1} / l
\end{array}\right. \\
& l=\frac{p_{\text {screw }} \theta_{m}}{2 \pi}
\end{aligned}
$$

where $l$ is the translation of the nut screw, $\theta_{m}$ is the ball scerw axis rotation angle and $p_{\text {screw }}$ is the pitch of the ball-screw axis.

Therefore the transmission ratio $\left(\tau_{\text {elbow }}\right)$ of the complete mechanism can be written as:

$$
\begin{gathered}
\dot{\gamma}=\dot{\alpha} \frac{a \cdot \sin (\alpha-\theta)}{c \cdot \sin (\gamma-\theta)} \quad \dot{\alpha}=-\frac{i}{b_{1} \cdot \sin \left(\alpha_{1}-\beta_{1}\right)} \\
\tau_{\text {ellow }}=\frac{\dot{\gamma}}{\omega_{m}}=\frac{p_{\text {screw }} a \cdot \sin (\alpha-\theta)}{2 \pi b_{1} c \cdot \sin (\gamma-\theta) \sin (\alpha-\beta)}
\end{gathered}
$$

$\tau_{\text {elbow }}$ expression shows that the mechanism is, by his nature, not homocinetic but the motion regularity can be improved by minimizing the following functions:

$$
F_{1}=\frac{\max (\tau)-\min (\tau)}{\max (\tau)} ; \quad F_{2}=\max \left|\frac{\partial \tau}{\partial \gamma}\right|
$$

obtaining, in this case, an average value of $\tau_{\text {elbow }}$ approximately of 0,0075 with a fluctuation within $15 \%$. Nevertheless the software of the elbow joint driver can take into account the dependency of $\tau_{\text {elbow }}$ by $\gamma$. 
The geometric parameters of the systems affect the transmission features and the size of the artificial joint. The influence of the variation of each single parameter can be analyzed separately (fig. 3) in order to choose the best fitting geometry of the system besides a tolerable fluctuation of the transmission ratio.

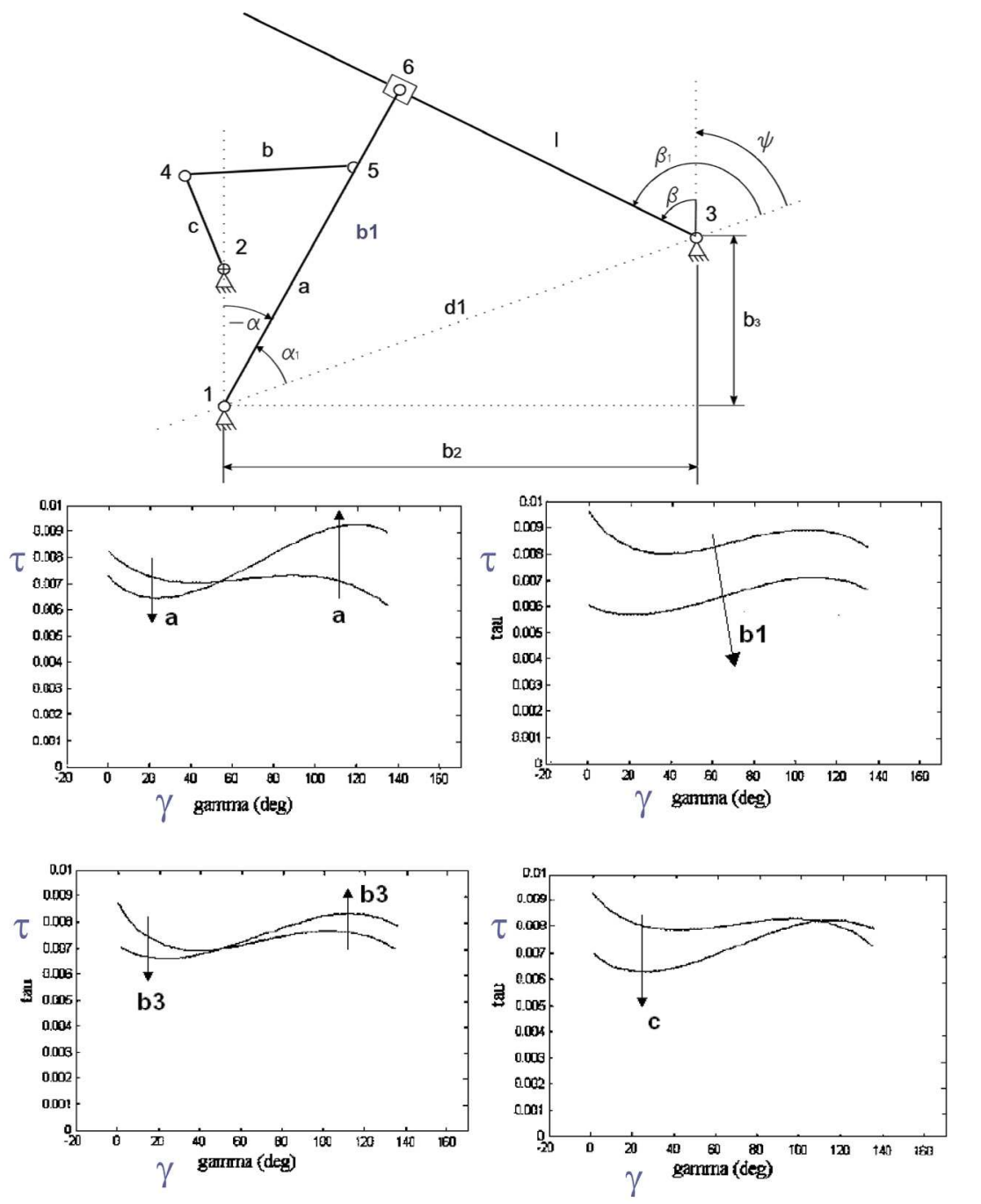

Fig. 6. Transmission ratio $\tau$ vs. elbow joint rotation $\gamma$ : influence of parameters $a, b_{1}, b_{3}, c$

Another important parameter that must be taken into account for a good design of bars linkages is the transmission angle, which is the lower angle between two consecutive bars. The optimum for this angle is $90^{\circ}$ but it varies during the mechanism motion: in general it must never assume values lower than $40^{\circ}$ in order to keep a good efficiency during the working phase and not to amplify the mechanism clearance (Magnani et al 1986). The 
following graphs (Fig. 7) show that the elbow joint, previously designed to fulfil the transmission ratio and the size requirement, is also within the limits for all the transmission angles of the linkage.
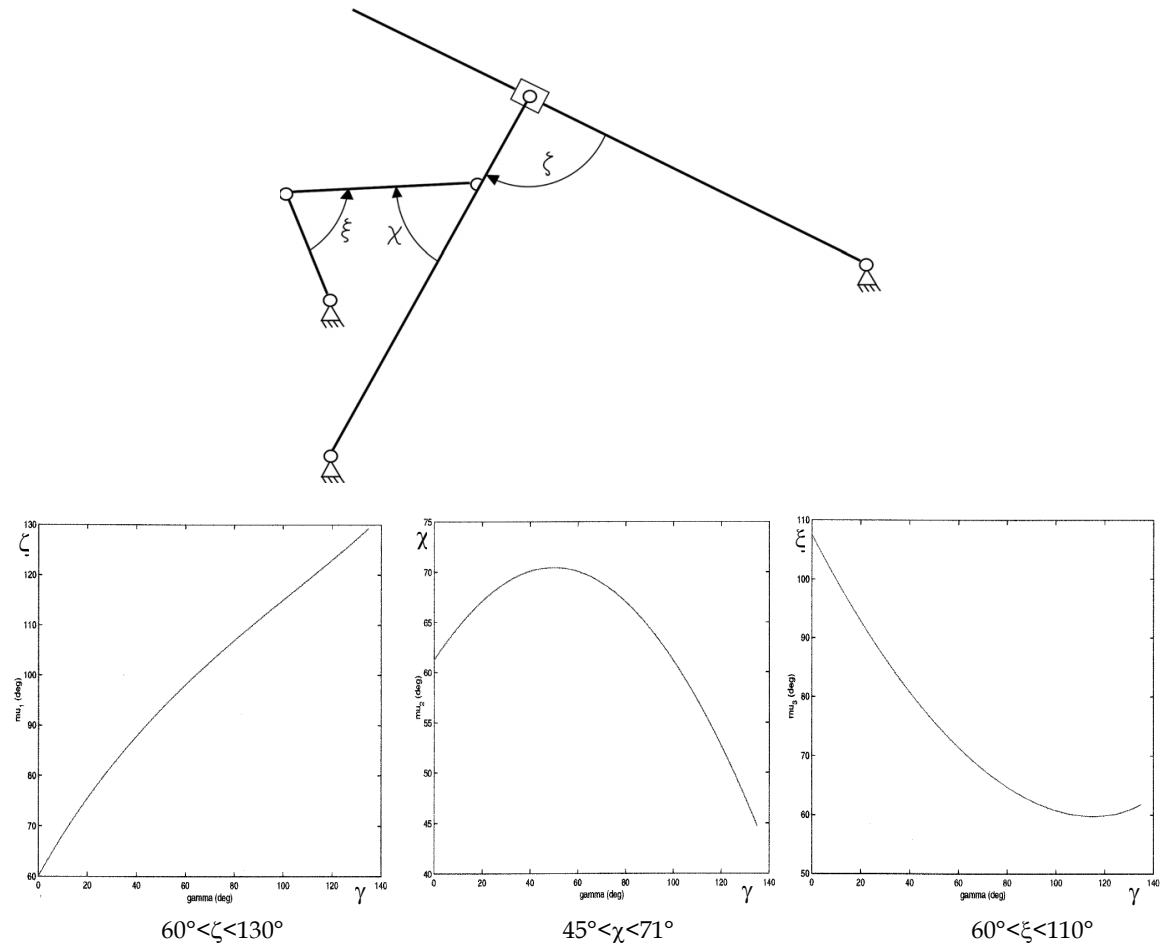

Fig. 7. transmission angles $\zeta, \chi$ and $\xi$ of the analyzed mechanism vs. the elbow rotation $\gamma$

\subsection{Extra d.o.f. for the artificial elbow}

A prosthetic arm for subjects disarticulated at the shoulder is, for the most advanced prototypes, equipped with an active shoulder joint with two d.o.f.(Fig.3 d, e). Therefore, despite the natural humero-ulnar joint is almost a perfect revolute pair, a prosthetic elbow could benefit of an extra d.o.f. located proximally on the forearm and in series to the elbow flexion, in order to replace the effects of the lacking axial rotation of the humerus. This new active axis, which increases the weight and the system complexity, is only convenient for subjects with head and trunk limited mobility.

Only for some specific tasks of the arm, such as drinking, for which the hand orientation is important for the whole cup trajectory, the subject can benefit of the extra axis. The following figure shows that the extra axis (fig.5, images on the right) allows to lead the cup to the mouth maintaining its symmetry axis perfectly vertical (fig.3, red dots), but they also show that, if a certain tilt is accepted (yellow $20^{\circ}$ and red dots $40^{\circ}$ ), it is also possible to drink with a full prosthetic arm mounting a single d.o.f. elbow(fig.5, images on the left). Furthermore, subjects with a good trunk mobility can easily adjust their head position to optimize the action. 
HAND WORKING VOLUME

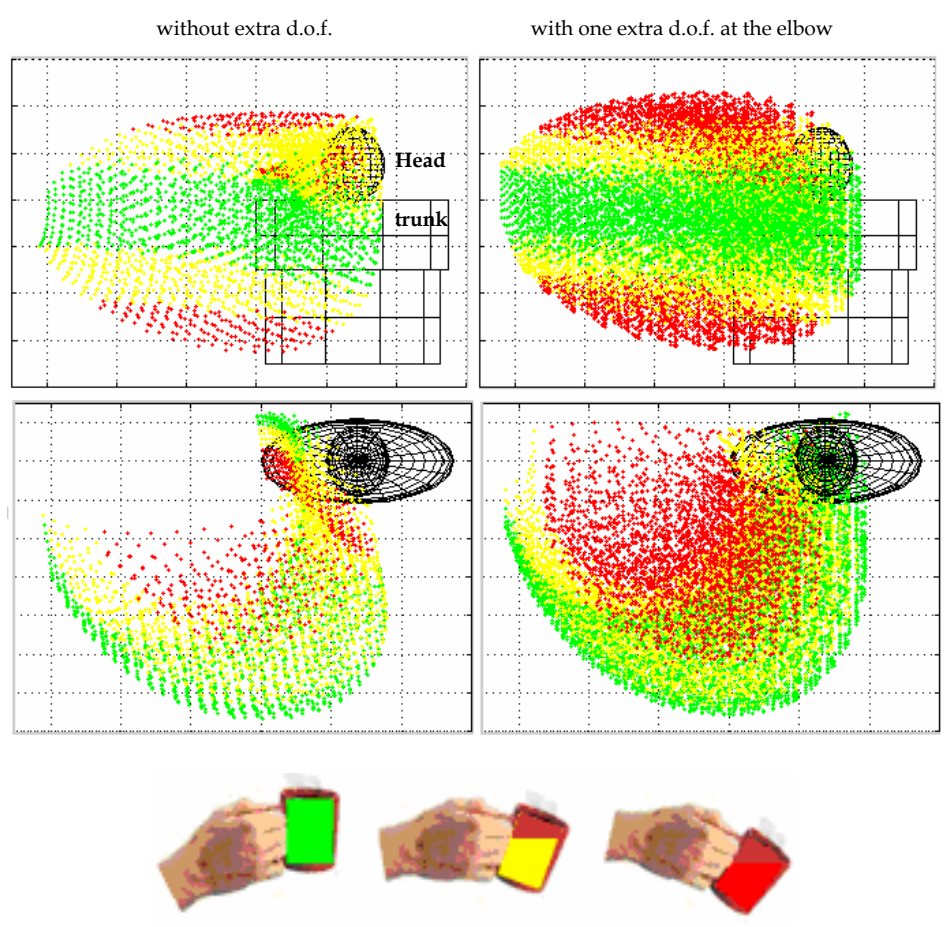

Fig. 5. Drinking task example: minimum inclination of a cup directed to the mouth following different trajectories for an artificial limb with two d.o.f. at the shoulder and one d.o.f. (left column) or two d.o.f. at the elbow (right column).

\section{Conclusion}

Externally powered prosthesis design requires preliminarily to solve the communication problem between the subject and the system: BCI is a promising technology but until now other signals collected for instance from head motion are used for driving purposes. Our research group designed modular systems for the whole upper limb replacement whose elbow can also be used separately for a transhumeral prosthetic arm. One approach to the design and the analysis of the performances of an electrically powered joint has been clarified referring to a real elbow joint developed by our group. Next improvement will concern the implementation of a recently developed mini hydrostatic transmission.

\section{References}

Camposaragna, M.; Casolo, F.; Cattaneo, B.; Lorenzi, F.(2001). Development of a computer simulation tool to design the motion control strategy of total artificial arm, Computer simulation in Biomechanics (p.165-172), Libreria Clup, ISBN887090, Milan 
Chilress, D.S.; Weir, R.F.; Craig, W; Heckathorne, M.D. (2001). Cineplasty as a control input for externally powered prosthetic components. J. of Rehabilitation Research and Development, Vol.38, No.4, (July/August 2001)

Magnani, P.L ; Ruggieri G. (1986). Meccanismi per machine automatiche. UTET, ISBN8802039348, Milan

Legani, G.; Casolo, F.; Righettini, P.; Zappa, B.(1996). A homogeneous matrix approach to 3D kinematics and dynamics - part 2: Applications to chains of rigid bodies and..., Mechanism E machine theory, vol. 31, no5, ISSN0094-114X Elsevier, Oxford

Nxp, B.V. (2007) I2C bus specification \& user manual rev.03, June 2007 ed.NSP, the Netherlands 


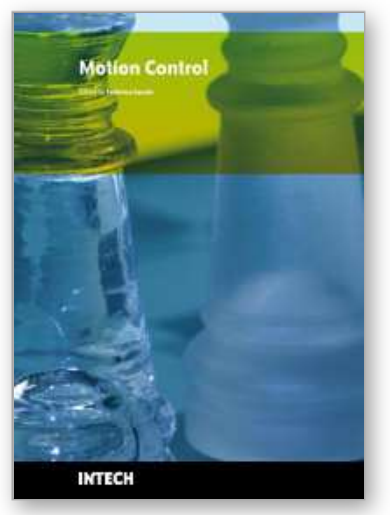

\author{
Motion Control \\ Edited by Federico Casolo
}

ISBN 978-953-7619-55-8

Hard cover, 590 pages

Publisher InTech

Published online 01, January, 2010

Published in print edition January, 2010

The book reveals many different aspects of motion control and a wide multiplicity of approaches to the problem as well. Despite the number of examples, however, this volume is not meant to be exhaustive: it intends to offer some original insights for all researchers who will hopefully make their experience available for a forthcoming publication on the subject.

\title{
How to reference
}

In order to correctly reference this scholarly work, feel free to copy and paste the following:

Federico Casolo (2010). Elbow Prosthesis for Partial or Total Upper Limb Replacements, Motion Control, Federico Casolo (Ed.), ISBN: 978-953-7619-55-8, InTech, Available from:

http://www.intechopen.com/books/motion-control/elbow-prosthesis-for-partial-or-total-upper-limb-replacements

\section{INTECH}

open science | open minds

\section{InTech Europe}

University Campus STeP Ri

Slavka Krautzeka 83/A

51000 Rijeka, Croatia

Phone: +385 (51) 770447

Fax: +385 (51) 686166

www.intechopen.com

\section{InTech China}

Unit 405, Office Block, Hotel Equatorial Shanghai

No.65, Yan An Road (West), Shanghai, 200040, China

中国上海市延安西路65号上海国际贵都大饭店办公楼405单元

Phone: +86-21-62489820

Fax: +86-21-62489821 
(C) 2010 The Author(s). Licensee IntechOpen. This chapter is distributed under the terms of the Creative Commons Attribution-NonCommercialShareAlike-3.0 License, which permits use, distribution and reproduction for non-commercial purposes, provided the original is properly cited and derivative works building on this content are distributed under the same license. 